\title{
Tuning Entanglement Patterns in Qubits Clusters
}

\author{
F. B. M. Dos Santos ${ }^{1}$, A. M. S. Macêdo ${ }^{2}$ \\ ${ }^{1}$ Departamento de Física, Universidade Estadual de Feira de Santana, Feira de Santana, Brasil \\ ${ }^{2}$ Laboratório de Física Teórica e Computacional, Departamento de Física, Universidade Federal de Pernambuco, Recife, Brasil \\ Email: fr_braz@yahoo.com.br
}

Received March 5, 2013; revised May 3, 2013; accepted May 20, 2013

Copyright (C) 2013 F. B. M. dos Santos, A. M. S. Macêdo. This is an open access article distributed under the Creative Commons Attribution License, which permits unrestricted use, distribution, and reproduction in any medium, provided the original work is properly cited.

\begin{abstract}
We identify patterns of ground state entanglement, or quantum discord, in qubit clusters with three and four qubits, that are induced by varying the couplings between next-nearest neighbors in the clusters. We show that these entanglement patterns can be associated with continuous multiply connected regions in parameter space, on which entanglement quantifiers, such as the pairwise concurrence, exhibit a particular type of behavior as a function of the couplings between next-nearest neighbors in the cluster. We present the distinct patterns in diagrams in parameter space with continuous boundary lines and we associate each pattern to a specific type of pure quantum correlation. We propose this procedure as a simple method to identify useful classes of pure quantum correlations in qubit networks.
\end{abstract}

Keywords: Entanglement; Patterns; Qubits

\section{Introduction}

Since the beginning of quantum mechanics, entanglement has been studied as a fundamental concept setting the main difference between classical and quantum mechanics $[1,2]$. Later, its study was intensified with the discovery that it is a valuable resource in quantum information protocols [3], such as quantum teleportation, dense coding and quantum cryptography [4,5]. In the last few years, notable results have shown that entanglement can be observed on a macroscopic scale [6] and can be a useful tool in the study of quantum phase transitions in condensed matter physics [7]. In particular, several works have analyzed scaling properties of ground state entanglement in spin chains in the proximity of quantum critical points of spin models, such as $X Y$ [8-11] and LMG [12-14] in the thermodynamic limit. The similarities of entanglement behavior in different critical systems suggested that it could lead to new insights in the study of quantum phase transitions and thus deepen the very notion of order in quantum systems [15]. Although the concept of quantum phase transition does not apply to finite systems, many works have analyzed the behavior of entanglement in finite clusters of quantum spins [16-18,20] and have shown that entanglement in these systems is significantly modified near special parameter values. These results could be particularly relevant for applications in quantum computation, where due to technolo- gical limitations the state of art is to realize it with only a few qubits [21-23].

For the purposes of implementing quantum computation, the physical system can be treated as a qubit network in which the couplings between the qubits can be controlled externally. The concept of qubit networks, on the other hand, can be related to that of quantum graphs, in which a quantum particle is bound to move through connectors and nodes in a network. This relation was analyzed in detail in [24], where it was described how to construct Hamiltonians for quantum walks, and how to map them onto Hamiltonians of qubit networks. An important advantage of this approach is that it provides a natural way to introduce decoherence and dissipation in the system. The ability to control the couplings implies the possibility to generate distinct topologies in the qubit network. It is well known, e.g., that a coupling between two subsystems is necessary for bipartite entanglement, but the precise relationship between the type of entanglement and the distribution of coupling strengths in the network can be strongly dependent on external parameters, such as applied magnetic fields and temperature [25]. In this context, systematic studies of the relationship between the amount and nature of entanglement and the topology of the network has been pursued in order to identify optimal topologies to create specific types of entanglements [26,27]. 
The distinction between classical and pure quantum correlations in general quantum states is a central question in quantum information theory. The subject has attracted much attention since the discovery that bipartite mixed separable states can have non-classical correlations that can be captured by a measure, denoted quantum discord [28], which equals the difference between two distinct definitions of mutual quantum information. For bipartite pure states quantum discord is equal to entanglement, but they can be very different for mixed states [29]. Recent results [30] suggest that the efficiency of a mixed state in a quantum computation depends fundamentally on its quantum discord. A rather different question is whether entanglement or quantum discord measures could be used to distinguish and classify distinct patterns of pure quantum correlations in qubit networks. The importance of this question can be seen, e.g., in the relationship between the notion of quantum order and the observation of distinct patterns of quantum entanglement in thermodynamically large systems [15,31]. Quantum orders emerge from non-local quantum correlations that sustain an ordered pattern without breaking any symmetry, and thus they cannot be described by Landau's theory of phase transitions. This led to the concept of topological order, defined as a pattern with long-range entanglement. More recently, a striking application of such concepts led to a full classification of symmetryprotected topological phases, defined as short-range-entangled quantum phases with a symmetry [32].

In a recent paper [19], it was shown that even in a finite system, where the notion of quantum order, defined via singularities in the energy density, does not apply, it is still possible to distinguish different types quantum correlations via measures of ground-state and thermal entanglements. These "entanglement patterns" were identified with classes of qualitatively distinct behaviors of the concurrency as a function of a control parameter. Interestingly, the set of parameter points associated with the same entanglement pattern forms a continuous multiply connected region in parameter space. Furthermore, it was shown how to construct pure quantum correlation functions that are highly sensitive to parameter changes that switch entanglement patterns. We stress that each entanglement pattern is associated with a particular type of quantum correlation and its role in quantum information theory could be paralleled with that of phases in the theory of phase transitions. With this in mind, one can argue that in order to optimally perform a specific quantum information process in a finite-size quantum network, some control parameters might need to be tuned for the selection of a particular pattern of quantum entanglement. As shown in Ref. [19], the choice of such tunings can be guided by means of a diagram in parameter space containing boundary lines that separate the different entan- glement patterns in the system. Understanding the role of the network topology in the emergence and tuning of such patterns and its association with different types of pure quantum correlations is one of the central motivations of the present study.

In this paper, we identify ground-state entanglement, or quantum discord, patterns in qubit clusters by analyzing the behavior of pairwise concurrences as a function of a control parameter that changes the coupling between certain pairs of qubits in the cluster, which ultimately leads to a change in the cluster topology. In Section 2, we classify entanglement patterns generated by gradually introducing an $X Y$ coupling between extremal qubits in an $X Y$ trimer, leading to a triangle configuration. This additional interaction monotonically increases the pairwise concurrence between the extremal qubits, but it also leads to a non-trivial behavior for the nearest-neighbor concurrence, $C(1)$. Our results show the existence of multiply connected regions in parameter space, whose boundary lines separate different types of entanglement patterns, which are characterized by different classes of behavior of the pairwise concurrences as a function of the variable coupling. We observe in particular an entanglement pattern, associated with a non-monotonic behavior of the nearest-neighbor concurrence, $C(1)$, with a maximum at an intermediate value of the coupling between the extremal qubits. In addition, we study for each entanglement pattern, the behavior of global entanglement, $Q$ and its part stored in pairwise entanglement, $Q_{p}$, as a function of the same control parameter. We find that although $Q$ always increases with the additional interaction, it also reduces $Q_{p}$ for weak magnetic fields, thus showing that the new coupling not only induces more global entanglement, but also modifies its nature. In the Section 3, we consider clusters with four qubits. The extremal limits, which are interpolated by additional coupling constants, are the nearest-neighbor $X Y$ square and the $L M G$ tetrahedron, where all qubits are homogeneously coupled. In this case, two pairs of next-nearest neighbor interactions are introduced via the transition from $X Y$ to $L M G$ model, which leads to a very rich diagram of entanglement patterns. We find that the nextnearest neighbor concurrence, $C(2)$, does not necessarily increase with the transition from $X Y$ to $L M G$ model. Consequently, the behavior of $C(2)$ also defines a set of regions in parameter space that can be associated with different entanglement patterns. For both types of pairwise concurrence, $C(1)$ and $C(2)$, we have regimes of monotonic increase, monotonic decrease and nonmono-tonic behavior, with a maximum at an intermediate value of the additional coupling parameter, $m$. A remarkable result in this case is the existence of a region between two limiting curves where $C(1)=C(2)$ for all values of $\mathrm{m}$. This means that in spite of the fact that the 
coupling constants between pairs of qubits are not all equal, the pairwise entanglement is equally distributed among all pairs of qubits. A summary and conclusions are presented in Section 5.

\section{Entanglement Patterns in Clusters with Three Qubits}

We analyze the effect of introducing a new coupling in a three qubit cluster with XY nearest-neighbor interactions, performing a change of topology: from the trimer topology, where the two extremal qubits are not coupled, to triangle topology, where all pairs of qubits interact equally. The Figure 1 presents these two reference topologies.

The goal this work is analyzing the modification on the amount and nature of the ground-state entanglement which results of the changing of topology. In order to perform this analyzes, we use an interpolating model where the coupling between extremal qubits is adjustable with a simple control parameter. The $X Y$ Hamiltonians which interpolate the topologies is:

$$
\begin{aligned}
H & -\frac{1}{2} \sum_{i=1}^{2}\left[(1+\gamma) \sigma_{i}^{x} \sigma_{i+1}^{x}+(1-\gamma) \sigma_{i}^{y} \sigma_{i+1}^{y}\right] \\
& -\frac{m}{2}\left[(1+\gamma) \sigma_{1}^{x} \sigma_{3}^{x}+(1-\gamma) \sigma_{1}^{y} \sigma_{3}^{y}\right]-b \sum_{i=1}^{3} \sigma_{i}^{z},
\end{aligned}
$$

where $\gamma$ is the anisotropy parameter, $b$ is the magnetic field. The operators $\sigma_{i}^{\alpha}$ are the Pauli's operators corresponding to the direction $\alpha$. The parameter $m$ controls the next-nearest neighbor coupling.

In matrix representation, we have a block diagonal matrix $H=[H(b), H(-b)]$ corresponding respectively to the canonical basis $B_{1}=\{|000\rangle,\langle 101|| 110\rangle,,|011\rangle\}$ and $B_{2}=\{|111\rangle,\langle 010|| 001\rangle,,|100\rangle\}$. The matrix elements of the two blocks are transformed onto one another by the change $b \rightarrow-b$. Thus, by determining the eigenvalues and eigenvectors of one of these $4 \times 4$ matrices, we can determine the ground-state $\left|\Psi_{g}\right\rangle$ and thus the entanglement. We restrict our analysis to the anti-ferromagnetic case.

Beyond the degeneracy at $b=0$, there is a special field value $b_{c}$, which depends on the anisotropy parameter, where the ground state is degenerate. In Figure 2, we show the degeneracy curves $b_{c}(\gamma)$ corresponding to the

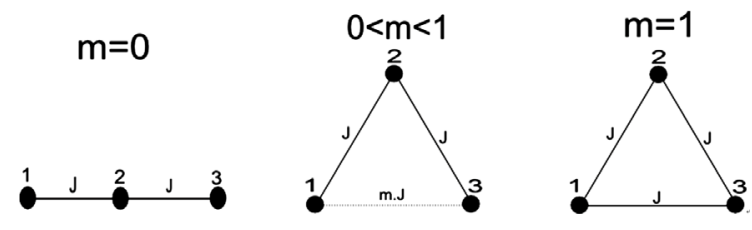

Figure 1. Qubit clusters in trimer, triangle and intermediate configurations.

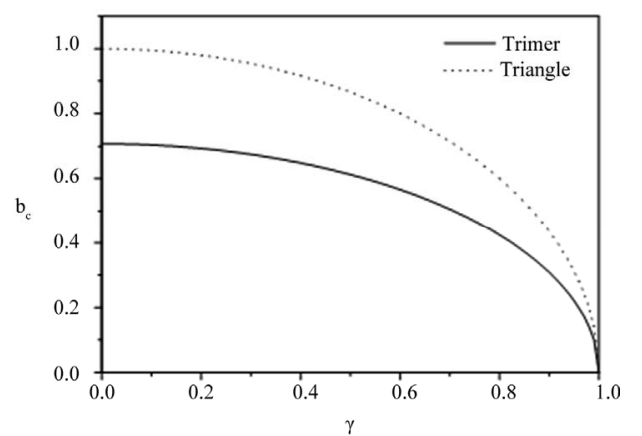

Figure 2. Critical fields $b_{c}$ for the triangle (solid) and trimer (dots) configurations as a function of the anisotropy parameter $\gamma$.

two configurations, trimer and triangle. For the triangle the special field value can be written as $\left|b_{c}\right|=\sqrt{1-\gamma^{2}}$. These two degeneracy curves separate three regions in parameters space, which will be important in the analysis of the behavior of the pairwise concurrences as a function of the coupling between the extremal spins.

In order to calculate the pairwise concurrence, we need to obtain the reduced density matrix of two selected qubits. In the triangle configuration, due to translation invariance, the reduced density matrices and, consequently, the pairwise concurrences, of every pair of qubits are the same. However, in the trimer configuration, we have two different types of two-qubit reduced density matrix $\rho_{i j}$, which can be written in the following form:

$$
\rho=\left(\begin{array}{llll}
u & 0 & 0 & w \\
0 & x & z & 0 \\
0 & z & y & 0 \\
w & 0 & 0 & v
\end{array}\right),
$$

where $u, v, w, x, y$ and $z$ are functions of $b$ and $\gamma$.

The concurrence can be calculated using Wooters' Formula [33] $C_{i j}=\max \left[0.2 \lambda^{\max }-\sum_{i} \lambda_{i}\right]$, where $\left\{\lambda_{i}\right\}$ are the square roots of eigenvalues of the non-hermitean matrix $R_{i j}=\rho_{i j} \sigma^{y} \otimes \sigma^{y} \rho_{i j}^{*} \sigma^{y} \otimes \sigma^{y}$. Using the reduced density matrix (2), we obtain $\lambda_{1,2}=|w \pm \sqrt{u v}|$ and $\lambda_{3,4}=|z \pm \sqrt{x y}|$. We obtain the following expression for the pairwise concurrence:

$$
C_{i j}=2 \max [0, w-\sqrt{x y}, z-\sqrt{u v}]
$$

With this model we can analyze the behavior of the pairwise concurrences $C(1)$ and $C(2)$ as a function of the coupling parameter $m$. In Figure 3, we show that the next-nearest neighbor concurrence, $C(2)$, monotonically increases with $m$ for all values of $b$ and $\gamma$ outside the shaded region. In the shaded region, $C(2)$ has discontinuities at degeneracy points. The behavior of $C(1)$, on the other hand, is more complicated and we can identify regions in parameter space associated with dif- 


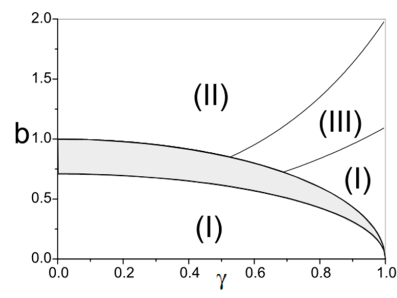

(a)

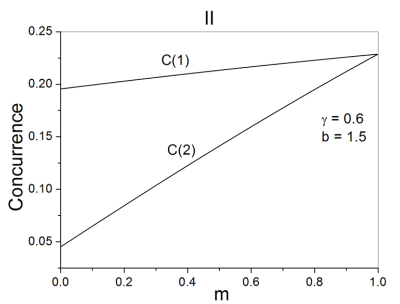

(c)

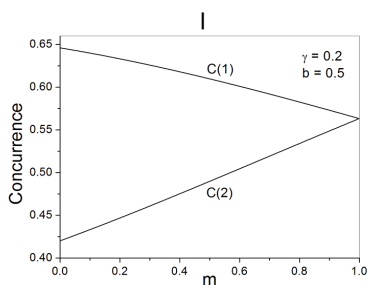

(b)

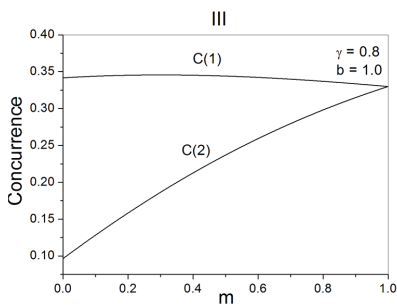

(d)
Figure 3. (a) Diagram $b \times \gamma$ with limiting lines separating the different patterns of entanglement classified by the behaviors of $C(1) \times \gamma$. Samples of the three distinct patterns are presented in (b)-(d). The shaded region is characterized by the coexistence of two different patterns separated by a discontinuity at the value of $\boldsymbol{m}$ where $b=b_{c}(\boldsymbol{m}, \gamma)$.

ferent patterns of entanglement, which are characterized by the shape of $C(1)$ as a function of $m$. Varying $m$ in the interval $[0,1], C(1)$ monotonically decreases in the region denoted (I) in Figure 3, and it increases in region (II). The region (III) is more interesting because $C(1)$ is non-monotonic and reaches a maximum at an intermediate value of $m$, which depends on the applied magnetic field. We stress that the anisotropy has an important hole in the emergence of the entanglement patterns. Note that for $\gamma$ lower than 0.5, approximately, we have only patterns associated with monotonic behavior of $C(1)$, while for $\gamma$ greater than 0.2 , we have the interesting additional pattern (III). For $\gamma>0.7$, approximately, we observe the resurgence of pattern (I) in the region of fields exceeding the special point $b_{c}$ of the triangle. The shaded region is characterized by the coexistence of two different patterns separated by a discontinuity at the value of $m$ where $b=b_{c}(m, \gamma)$. As example, the Figure 4 present the behavior of $C(1) \times m$ for $\gamma=0.4$ and $b=0.8$. As we can see, the region does not correspond to a new pattern of entanglement, but a region of coexistence of two patterns.

\section{Changing the Nature of Entanglement}

In this subsection we analyze the possibility to transform entanglement stored in pairwise concurrences in global entanglement which has not this nature. As a quantifier of global entanglement, we use the Q-measure [34], which corresponds, for a cluster with N qubits,

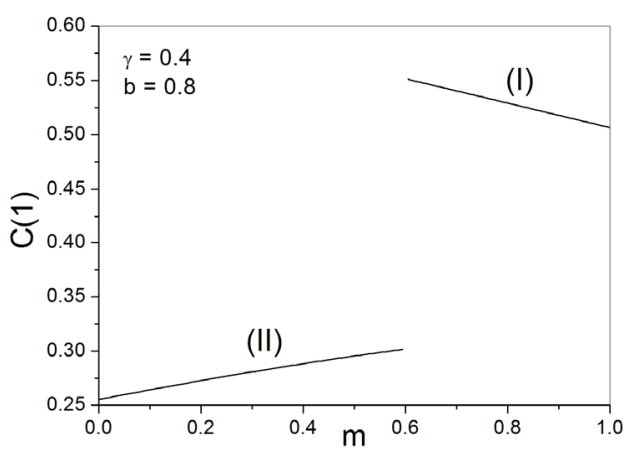

Figure 4. Sample of a graph in the intermediate region. The graph present a discontinuity in $m=0.6$ approximately where $b=b_{c}(m, \gamma)$, which separate the two distinct patterns, (I) e (II).

to the average purity of the reduced density matrices of each qubit

$$
Q=\sum_{i=1}^{N} 2\left[1-\operatorname{Tr}\left(\rho_{i}^{2}\right)\right]
$$

where $\rho$ is the reduced density matrix of the i-th qubit and $\mathrm{N}$ is the number of qubits. In the three qubit case, the reduced matrices of extremal qubits are identical $\rho_{1}=\rho_{3}$. Thus, the Q-measure reduces to $Q=(2 / 3)\left[3-2 \operatorname{Tr}\left(\rho_{1}^{2}\right)-\operatorname{Tr}\left(\rho_{2}^{2}\right)\right]$.

As we have seen, the pairwise concurrences can decrease or increase with the parameter $m$. However, the the global entanglement should be an increasing monotonic function of $m$, which is indeed what we observe in Figure 5.

The part of the global entanglement stored in pairwise concurrences can be calculated using the concept of distributed entanglement or monogamy of entanglement $[35,36]$. In this concept, the entanglement between a qubit and the others in the cluster is used as a bound for the amount of entanglement stored in pairwise concurrences. It is defined by the sum of squares of all pairwise concurrences involving that qubit. Thus, the part of global entanglement stored in pairwise concurrences can be estimated by the average of these sums over all qubits in the cluster, which we denote by $Q_{p}$. For a three-qubit cluster, we obtain $Q_{p}=(2 / 3)\left[2 C(1)^{2}+C(2)^{2}\right]$. In Figure 5 we show the behavior of $\mathrm{Q}$ and $Q_{p}$ as functions of the interaction parameter $m$. In all regions shown in the $b \times \gamma$ diagram, the global entanglement, $Q$, increases monotonically with $m$, but the part stored in pairwise entanglement, $Q_{p}$, exhibits two different behaviors. In regions (II) and (III), $Q_{p}$ increases with $m$, confirming that pairwise entanglement is created by changing the coupling. On the other hand, in region (I), $Q_{p}$ decreases monotonically with $m$, indicating that the additional coupling not only creates global entanglement 


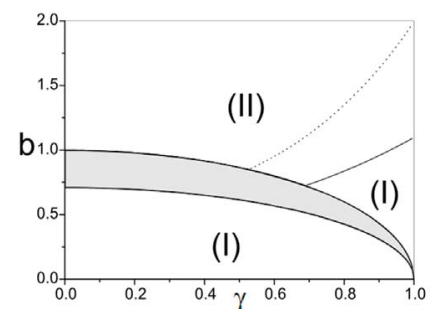

(a)

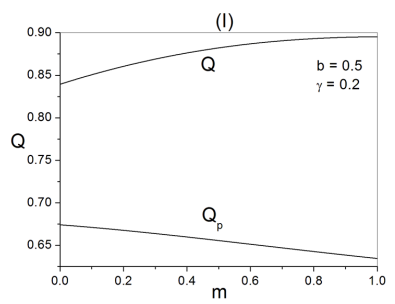

(b)

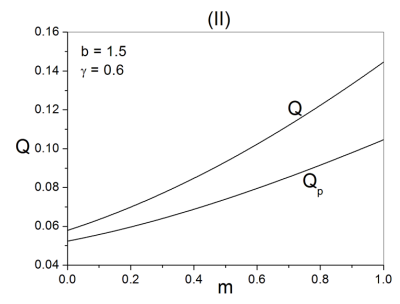

(c)
Figure 5. Global entanglement $Q$ and pairwise entanglement $Q_{p}$ in a three qubit cluster with a variable coupling parameter $\boldsymbol{m}$. (a) Diagram $b \times \gamma$ with regions corresponding to different entanglement patterns obtained from the pairwise concurrence $C(1)$. (b) Samples of the two distinct regions.

but also converts entanglement stored in pairs into genuine multipartite entanglement.

\section{Entanglement Patterns in Clusters with Four Qubits}

In this section we identify entanglement patterns in clusters with four qubits induced by the introduction of nextnearest neighbor interactions. The model systems are the Heisenberg \$XY\$ model with nearest neighbor interactions and the \$LMG\$ model with identical interactions between all pairs of qubits. The model Hamiltonian contains an interpolating parameter $m$, used to control the next-nearest neighbor interaction, and is given by

$$
\begin{aligned}
H & -\frac{1}{2} \sum_{i=1}^{4}\left[(1+\gamma) \sigma_{i}^{x} \sigma_{i+1}^{x}+(1-\gamma) \sigma_{i}^{y} \sigma_{i+1}^{y}\right] \\
& -\frac{m}{2} \sum_{i=1}^{2}\left[(1+\gamma) \sigma_{i}^{x} \sigma_{i+1}^{x}+(1-\gamma) \sigma_{i}^{y} \sigma_{i+1}^{y}\right]-b \sum_{i=1}^{4} \sigma_{i}^{z}
\end{aligned}
$$

In Figure 6, we show all configurations described by the interpolating Hamiltonian $H$. The square configuration is obtained by setting $m=0$ in $H$, whilst the tetrahedron is obtained with $m=1$. Figure 7 presents the degeneracy fields for the two extremal configurations as functions of the anisotropy parameter $\gamma$. For $m=0$ we have two degeneracy fields, one that coincides with the degeneracy field of the three-qubit clusters, $b_{c}=\sqrt{1-\gamma^{2}}$, and a smaller one, obtained numerically by a non-linear

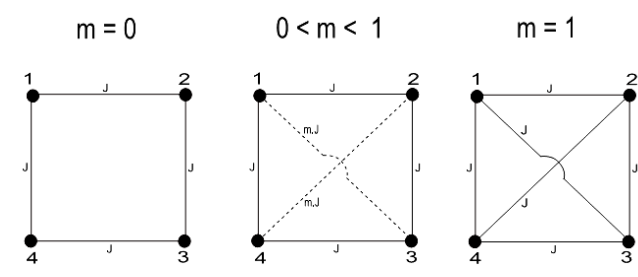

Figure 6. Topologies for four spin clusters. At left, square configuration with nearest neighbor only. At middle, interpolating configuration with next-nearest neighbor coupling, which is controlled by $m$. At right Tetrahedron configuration with interactions among all qubits.

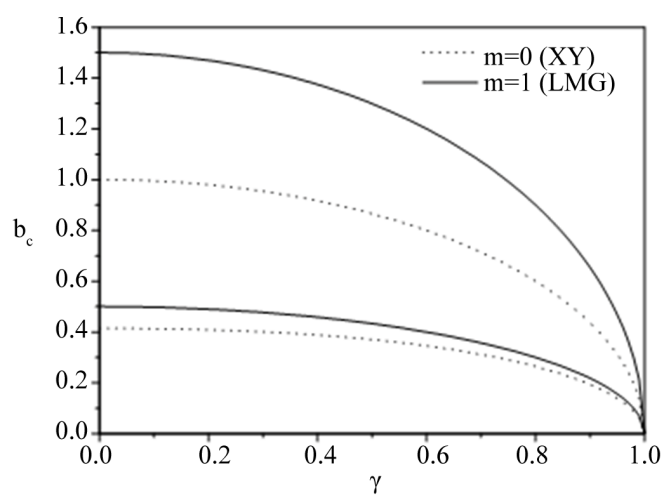

Figure 7. Degeneracy fields as functions of the anisotropy parameter. The solid (dotted) lines were obtained from the $X Y$ (LMG) model.

fit, which is given by $b_{c} \approx\left(0.414+0.056 \gamma^{2}\right) \sqrt{1-\gamma^{2}}$. For $m=1$, the two degeneracy fields have also been obtained numerically, but we found that they adjust well to sectors of the ellipses $b_{c}=1.5 \sqrt{1-\gamma^{2}}$ and $b_{c}=0.5 \sqrt{1-\gamma^{2}}$. The fact that there are two degeneracy curves as a function of the anisotropy parameter implies a much richer structure of entanglement patterns in comparison with the three qubits case.

Since we are not interested in the behavior of the pairwise concurrencies as functions of the magnetic field, we present in this section only results obtained by varying the interpolation parameter $m$. Although it is hard to obtain analytically the exact ground-state of the interpolating model, it is possible to calculate it explicitly in the intermediate region $0.5 \sqrt{1-\gamma^{2}}<b<\sqrt{1-\gamma^{2}}$. It has the form

$$
\begin{array}{r}
\left|\Psi_{G}\right\rangle=\sqrt{p_{m}}\left|W_{4}\right\rangle+\sqrt{1-p_{m}}\left|W_{4}\right\rangle \\
\sqrt{p_{m}}=\frac{2 b+\sqrt{4 b^{2}+\gamma^{2}(m+2)^{2}}}{\sqrt{\gamma^{2}(m+2)^{2}+\left(2 b+\sqrt{4 b^{2}+\gamma^{2}(m+2)^{2}}\right)^{2}}}, \\
\left|W_{4}\right\rangle=\frac{1}{2}[|0001\rangle+|0010\rangle+|0100\rangle+|1000\rangle] \\
\left|W_{4}\right\rangle=\frac{1}{2}[|1110\rangle+|1101\rangle+|1011\rangle+|0111\rangle] .
\end{array}
$$


The states $\left|W_{4}\right\rangle$ and $\left|W_{4}\right\rangle$ are known as $W$-states and have maximum multipartite entanglement and the pairwise concurrences in any of these states is invariant under pair exchange. The ground state in this region is a coherent superposition of these states and thus it preserves this invariance, which in turn explains the observed property $C(1)=C(2)$ for all values of $m$.

In order to analyze in more detail the effect of the additional couplings in the four-qubit cluster, we study the behavior of the concurrence as a function of the interacttion parameter $m$ in the interval $[0,1]$, for several values of the magnetic field and anisotropy parameter. For each value of $m$ we have two degeneracy fields, each bounded by a solid and a dotted line, shown in Figure 7.

We observe discontinuities in $C(1) \times \gamma$ for fields in the shaded regions shown in Figure 8 at the values of $m$ that satisfies the equation $b=b_{c}(m, \gamma)$. In these regions, two distinct entanglement patterns coexist.

A novel feature of the four qubits case is the fact that both $C(1) \times \gamma$ and $C(2) \times \gamma$ present qualitative changes as we move in parameter space, thus implying a very reach structure of entanglement patterns. In Figure 8 we present a diagram $b \times \gamma$, in which we separate regions corresponding to different entanglement patterns characterized by qualitative changes in the behavior of $C(1) \times \gamma$.

In the region denominated (I), $C(1)$ monotonically decreases with $m$. In region (II) we find that $C(1)$ monotonically increases, while in region (III), $C(1)$ is non-monotonic and exhibits a local maximum. In the Figure 9 we present the entanglement patterns characterized by the behavior of $C(2) \times \gamma$. In the regions denominated (I), $C(2)$ monotonically decreases with $m$. In (II), we find that $C(2)$ monotonically increases, while in region (III), $C(2)$ is non-monotonic and ex-

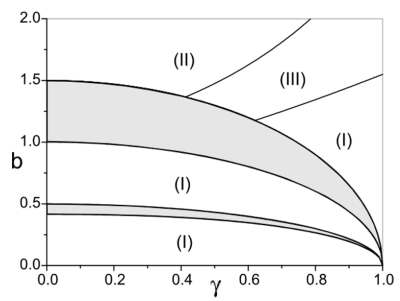

(a)

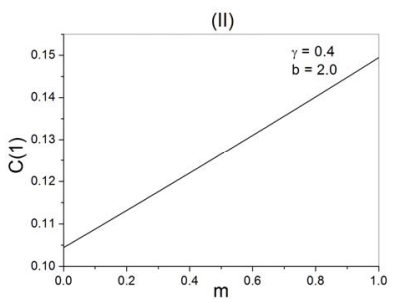

(c)

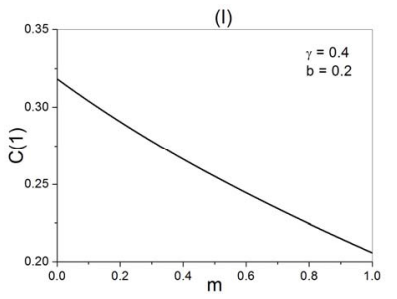

(b)

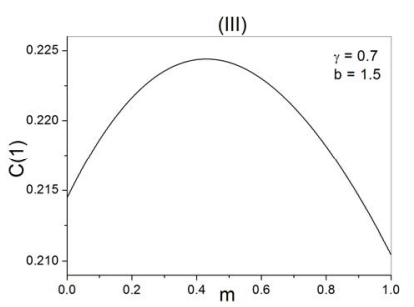

(d)
Figure 8. Upper part: Diagram $b \times \gamma$ showing boundary lines separating the different patterns of $C(1)$. The other graphs present samples of each region of the diagram. hibits a local maximum. Finally, in region (IV), we find a behavior that does not appear in $C(1)$ : the emergence of a finite interval of values of $m$, on which $C(2)=0$.

\section{Global Entanglement}

As in the three qubits case, we shall complete the analysis by describing the behavior of the global entanglement $Q$ and the pairwise-stored entanglement $Q_{p}$ as functions of the interpolation parameter $m$ in all regions associated with different entanglement patterns. In Figure 10 we present a diagram $b \times \gamma$ combining all regions

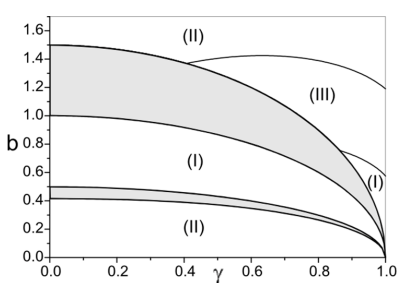

(a)

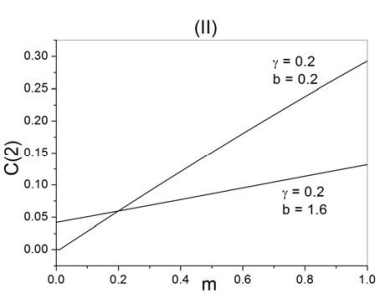

(c)

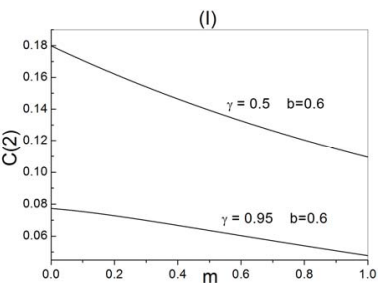

(b)

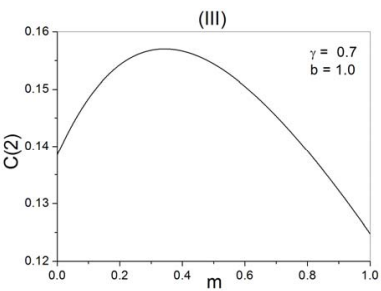

(d)
Figure 9. Upper part: Diagram $b \times \gamma$ showing boundary lines separating the different patterns of $C(2)$. The other graphs present samples of each region of the diagram.

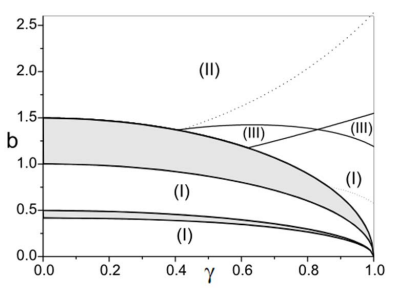

(a)

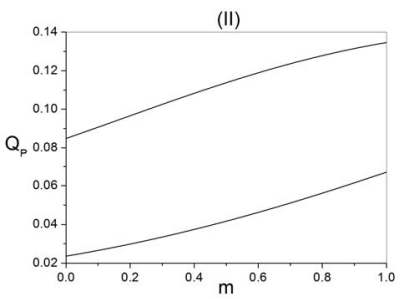

(a)

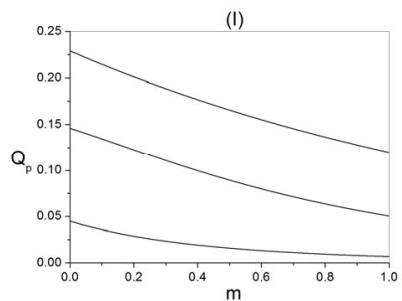

(b)

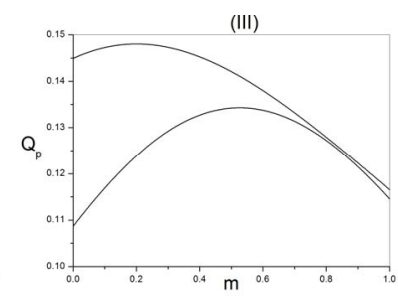

(b)
Figure 10. Upper part: Diagram $b \times \gamma$ showing boundary lines separating the different entanglement patterns. The dotted lines separate only patterns of $C(1)$ an $C(2)$. The behavior of $Q_{p} \times m$ defines the distinct patterns. 
with entanglement patterns characterized by the behaviors of $C(1) \times \gamma$ and $C(2) \times \gamma$.

We find basically three types of behaviors: A monotonic increase with $m$, which occurs in regions (I), a monotonic decrease in regions (II), and a non monotonic behavior with a local maximum in regions (III).

A notable behavior is observed in the regions (I), as the additional couplings always increases the global entanglement and the pairwise-stored entanglement decrease in this region, we can say that the additional couplins converts converts pairwise-stored entanglement in genuine multipartite entanglement. Remarkably, in region (III) this conversion occurs only for values of $\mathrm{m}$ greater than the one corresponding to the maximum of $Q_{p}$. This behavior illustrates the changing on the nature of entanglement, which occurs also in three spins chains.

\section{Conclusions}

In this paper we showed how to identify entanglement patterns in qubit clusters that are induced by varying the couplings between next-nearest neighbors in the cluster. The procedure consists in separating points in parameter space according to the distinct types of behavior of the nearest neighbor and next-nearest neighbor concurrences as a function of the variable coupling parameter. We applied this procedure to clusters with three and four qubits. We found that points in parameter space associated with qualitatively similar behavior of the pairwise concurrences form a continuous multiply connected region, thus allowing its association to a pattern of entanglement. The results were presented in diagrams with continuous boundary lines separating the distinct entanglement patterns.

There are several ways in which the information contained in the diagrams of entanglement patterns could be used in practical applications. As an example, consider a cluster of three qubits in the trimer configuration. Suppose that the task is to produce a certain amount of entanglement between the extremal qubits, measured by $C(2)$, by switching on an interaction between them, but without reducing the amount of pairwise entanglement with the other qubit, measured by $C(1)$. From the diagram and graphs in Figure 4, we see immediately that we need to tune the control parameters $b$ and $\gamma$ into region (II). If, on the other hand, we want to study the gradual transition from a regime in which both $C(1)$ and $C(2)$ increases to another one in which $C(1)$ decreases and $C(2)$ increases, then from Figure 4 we see that we need to tune $b$ and $\gamma$ into region III.

Clearly, as the cluster increases in size the diversity of entanglement patterns should also increase, thus making the full classification a very hard job. However, if a certain practical application requires that one concentrates on a particular type of pattern, finding its region in pa- rameter space is a much simpler task. Studying concrete examples of practical applications of entanglement patterns in quantum networks is an interesting perspective for further research.

\section{Acknowledgements}

This work was supported by CNPq and Capes (Brazilian Agency), and by UEFS and UFPE (Brazilian Universities).

\section{REFERENCES}

[1] A. Einstein, B. Podolsky and N. Rosen, "Can QuantumMechanical Description of Physical Reality Be Considered Complete?” Physical Review, Vol. 47, No. 10, 1935, pp. 777-780. doi:10.1103/PhysRev.47.777

[2] J. S. Bell, "On the Einstein Podolsky Rosem Paradox," Physics, Vol. 1, 1964, pp. 195-200.

[3] C. H. Bennett and D. P. DiVincenzo, "Quantum Information and Computation,” Nature, Vol. 404, pp. 247-255. doi:10.1038/35005001

[4] M. A. Nielsen and I. L. Chuang, "Quantum Computation and Quantum Information,” Cambridge University Press, Cambridge, New York, 2000.

[5] R. Horodecki, P. Horodecki, M. Horodecki and K. Horodecki, "Quantum Entanglement," Reviews of Modern Physics, Vol. 81, No. 2, 2009, pp. 865-942. doi:10.1103/RevModPhys.81.865

[6] L. Amico, R. Fazio, A. Osterloch and V. Vedral, "Entanglement in Many-Body Systems," Reviews of Modern Physics, Vol. 80, No. 2, 2008, pp. 517-576. doi:10.1103/RevModPhys.80.517

[7] S. Sachdev, "Quantum Phase Transitions," 2nd Edition, Cambridge University Press, New York, 2011. doi:10.1017/CBO9780511973765

[8] G. Vidal, J. I. Latorre, E. Rico and A. Kitaev, "Entanglement in Quantum Critical Phenomena," Physical Review Letters, Vol. 90, No. 22, 2003, Article ID: 227902. doi:10.1103/PhysRevLett.90.227902

[9] J. I. Latorre, E. Rico and G. Vidal, "Ground State Entanglement in Quantum Spin Chains,” Quantum Information and Computation, Vol. 4, 2004, pp. 48-92.

[10] T. J. Osborne and M. A. Nielsen, "Entanglement in a Simple Quantum Phase Transition,” Physical Review A, Vol. 66, No. 3, 2002, Article ID: 032110. doi:10.1103/PhysRevA.66.032110

[11] A. Osterloh, L. Amico, G. Falci and R. Fazio, "Scaling of Entanglement Close to a Quantum Phase Transition”, $\mathrm{Na}$ ture, Vol. 416, 2002, pp. 608-610. doi:10.1038/416608a

[12] J. Vidal, G. Palacios and R. Mosseri, "Entanglement in a Second-Order Quantum Phase Transition,” Physical Review A, Vol. 69, No. 2, 2004, Article ID: 022107. doi:10.1103/PhysRevA.69.022107

[13] J. I. Latorre, R. Orus, E. Rico and J. Vidal, “Entanglement Entropy in the Lipkin-Meshkov-Glick Model,” Physical Review A, Vol. 71, No. 6, 2005, Article ID: 064101. 
doi:10.1103/PhysRevA.71.064101

[14] S. Dusuel and J. Vidal, "Continuous Unitary Transformations and Finite-Size Scaling Exponents in the LipkinMeshkov-Glick Model,” Physical Review B, Vol. 71, No. 22, 2005, Article ID: 224420. doi:10.1103/PhysRevB.71.224420

[15] X.-G. Wen, "Quantum Field Theory of Many-Body Systems," Oxford University Press, Oxford, New York, 2004.

[16] G. L. Kamta and A. F. Starace, “Anisotropy and Magnetic Field Effects on the Entanglement of a Two Qubit Heisenberg XY Chain,” Physical Review Letters, Vol. 88, No. 10, 2002, Article ID: 107901. doi:10.1103/PhysRevLett.88.107901

[17] X. Wang, "Entanglement in the Quantum Heisenberg XY Model,” Physical Review A, Vol. 64, No. 1, 2001, Article ID: 012313. doi:10.1103/PhysRevA.64.012313

[18] S.-J. Gu, H. Li, Y.-Q. Li and H.-Q. Lin, "Entanglement of the Heisenberg Chain with the Next-Nearest-Neighbor Interaction,” Physical Review A, Vol. 70, No. 5, 2004, Article ID: 052302. doi:10.1103/PhysRevA.70.052302

[19] F. B. M. dos Santos, R. M. Dias and A. M. S. Macêdo, "Entanglement Patterns and Pure Quantum Correlations in the Heisenberg XY Model,” Physical Review A, Vol. 79, No. 3, 2009, Article ID: 032329. doi:10.1103/PhysRevA.79.032329

[20] K. Wu, B. Zhou and W. Cao, “Thermal Entanglement in a Four-Qubit Heisenberg Spin Model with External Magnetic Fields,” Physics Letters A, Vol. 362, No. 5-6, 2007, pp. 381-389. doi:10.1016/j.physleta.2006.10.045

[21] A. Imamoglu, D. D. Awschalom, G. Burkard, D. P. DiVincenzo, D. Loss, M. Sherwin and A. Small, "Quantum Information Processing Using Quantum Dot Spins and Cavity QED,” Physical Review Letters, Vol. 83, No. 20, 1999, pp. 4204-4207. doi:10.1103/PhysRevLett.83.4204

[22] J. I. Cirac and P. Zoller, “A Scalable Quantum Computer with Ions in an Array of Microtraps," Nature, Vol. 404, 2000, pp. 579-581. doi:10.1038/35007021

[23] Y. Makhlin, G. Schön and A. Shnirman, "JosephsonJunction Qubits with Controlled Couplings,” Nature, Vol. 398, 1999, pp. 305-307, doi:10.1038/18613

[24] A. P. Hines and P. C. E. Stamp, "Quantum Walks, Quantum Gates, and Quantum Computers”, Physical Review A, Vol. 75, No. 6, 2007, Article ID: 062321. doi:10.1103/PhysRevA.75.062321
[25] M. C. Arnesen, S. Bose and V. Vedral, "Natural Thermal and Magnetic Entanglement in the 1D Heisenberg Model," Physical Review Letters, Vol. 87, No. 1, 2001, Article ID: 017901. doi:10.1103/PhysRevLett.87.017901

[26] A. Hutton and S. Bose, "Comparison of Star and Ring Topologies for Entanglement Distribution,” Physical Review A, Vol. 66, No. 3, 2002, Article ID: 032320. doi:10.1103/PhysRevA.66.032320

[27] A. Hutton and S. Bose, "Mediated Entanglement and Correlations in a Star Network of Interacting Spins,” Physical Review A, Vol. 69, No. 4, 2004, Article ID: 042312. doi:10.1103/PhysRevA.69.042312

[28] H. Ollivier and W. Zurek, "Quantum Discord: A Measure of the Quantumness of Correlations," Physical Review Letters, Vol. 88, No. 1, 2001, Article ID: 017901. doi:10.1103/PhysRevLett.88.017901

[29] T. Werlang and G. Rigolin, "Thermal and Magnetic Quantum Discord in Heisenberg Models”, Physical Review A, Vol. 81, No. 4, 2010, Article ID: 044101. doi:10.1103/PhysRevA.81.044101

[30] A. Datta, A. Shaji and C. M. Caves, "Quantum Discord and the Power of One Qubit," Physical Review Letters, Vol. 100, No. 5, 2008, Article ID: 050502. doi:10.1103/PhysRevLett.100.050502

[31] X.-G. Wen, "Quantum Orders in an Exact Soluble Model,” Physical Review Letters, Vol. 90, No. 1, 2003, Article ID: 016803. doi:10.1103/PhysRevLett.90.016803

[32] X. Chen, Z.-C. Gu, Z.-X. Liu and X.-G. Wen, "Symmetry Protected Topological Orders and the Group Cohomology of Their Symmetry Group,” arXiv:cond-mat/1106.4772, 2012.

[33] W. K. Wootters, "Entanglement of Formation of an Arbitrary State of Two Qubits," Physical Review Letters, Vol. 80, No. 10, 1998, pp. 2245-2248. doi:10.1103/PhysRevLett.80.2245

[34] D. A. Meyer and N. R. Wallach, "Global Entanglement in Multiparticle Systems," Journal of Mathematical Physics, Vol. 43, No. 9, 2002, p. 4273. doi:10.1063/1.1497700

[35] V. Coffman, J. Kundu and W. K. Wootters, "Distributed Entanglement,” Physical Review A, Vol. 61, No. 5, 2000, Article ID: 052306. doi:10.1103/PhysRevA.61.052306

[36] T. J. Osborne and F. Verstraete, "General Monogamy Inequality for Bipartite Qubit Entanglement," Physical Review Letters, Vol. 96, No. 22, 2006, Article ID: 220503. doi:10.1103/PhysRevLett.96.220503 